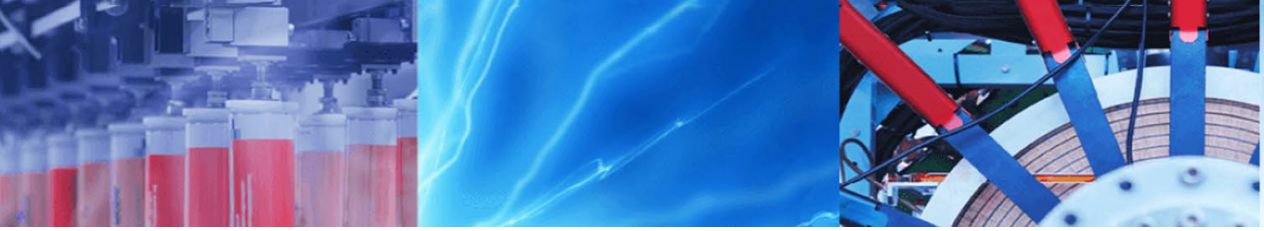

Research Article

\title{
Passive flow control mechanism in a bio-inspired corrugated hydrofoil
}

\author{
P. Sooraj ${ }^{1} \cdot$ Amit Agrawal $^{1}$ (D)
}

Received: 2 August 2019 / Accepted: 22 October 2019 / Published online: 28 October 2019

(c) Springer Nature Switzerland AG 2019

\begin{abstract}
Corrugated hydrofoils are lately getting attention because of their superior aerodynamic performance compared to engineered hydrofoils at low Reynolds numbers (Re). A particle image velocimetry (PIV) based study on corrugated hydrofoil is conducted here to understand the flow dynamics around it at ultralow Reynolds numbers $(\operatorname{Re}=280-11,700)$. Seven different angles of attack $(a)$ are considered in this study ranging from $-15^{\circ}$ to $15^{\circ}$. Load cell measurements are undertaken to obtain the force coefficients and further these are compared with the results obtained from PIV data using wake survey method. The wake velocity profiles are examined to understand the variation in force coefficients in a better way. Vortices are found to be trapped in the valley of the corrugations. The lift coefficient increases as the number of vortices increases on the top (suction) surface. A temporal analysis of the data shows that the partially merged co-rotating vortices give higher lift as compared to the fully merged vortices. The maximum aerodynamic performance is obtained at $-5^{\circ}$ angle of attack for $R e=6760$. The asymmetry in the geometry combined with asymmetry in the flow helps create relatively high lift for a corrugated wing. The performance of positive and negative angles of attack are compared and it is found that the fluctuation in lift coefficient is comparatively higher. It is hypothesized that the merging of trapped vortices with each other gives the effect of fluid roller bearings; this fluid roller bearing produces a travelling wave, which avoids the formation of boundary layer, thereby leading to high gliding ratio. These detailed results, covering the entire Reynolds number and angle of attack range of dragonfly flight, provide useful insights into the secret of dragonfly flight which will help in better design of micro air vehicles.
\end{abstract}

Keywords Corrugated hydrofoil · Load cell · Vortex merging · Lift coefficient · Drag coefficient

\section{Introduction}

The real time measurements on dragonfly wings showed that the lift production is higher compared to other insects $[2,42]$. Wakeling and Ellington [42] suggested that the increased lift generation is not because of the absolute wing area, aspect ratio or Reynolds number, rather due to the corrugations in the wing cross section. Kesel [11] also observed that the corrugations in the wing cross section play an important role towards the production of high lift. Kesel studied the variation of force coefficients for three different corrugated profiles at Reynolds numbers $7880-10,000$ and angles of attack $-25^{\circ}-40^{\circ}$. Hu and
Tamai [10] noticed that corrugated airfoil has better aerodynamic performance than smooth airfoils at $\operatorname{Re}=34,000$. Also, corrugated airfoil delays large scale flow separation thereby delaying stall. They reported force coefficient data obtained using force-moment sensor. Sooraj and Agrawal [36] experimented in the Reynolds number range of 340-11,700 and found that the shape of the corrugation plays an important role in producing vortices. Interestingly, they reported that the shape of the virtual hydrofoil produced around the corrugated airfoil is similar to that of Prandtl D-root ns airfoil for negative angle of attack.

The dragonflies glide frequently during their flight [42]. According to Heinrich [9] and May [21, 22], the gliding

Amit Agrawal, amit.agrawal@iitb.ac.in|'Department of Mechanical Engineering, Indian Institute of Technology Bombay, Powai, Mumbai 4000076, India. 
ability is used for thermoregulatory actions of their body by convective cooling; moving through the air without extra heat production. May [20-22], Corbet [6] and Polcyn [31] noticed that the frequency of gliding increases as the ambient temperature increases. Therefore, gliding flight is inevitable and important for dragonflies during their flight. Sunada et al. [38] noticed that the aerodynamic performance is better at low Reynolds number for a sharp leading edge. Murphy and $\mathrm{Hu}$ [29] suggested that the superior aerodynamic performance of the corrugated airfoil is due to the protruding corners which enhances the laminar to turbulent transition while the flow is attached to the virtual airfoil profile. The trapped vortices further help to make the boundary layer more energetic by pulling the high-speed flow near to the corrugated profile. They used force sensor measurement in their study and also compared the lift coefficient obtained from the potential flow theory for Reynolds number range $5.8 \times 10^{4}-1.25 \times 10^{5}$ and angle of attack $0^{\circ}-20^{\circ}$. The potential flow assumption was reported to be getting violated at higher angles of attack and higher Reynolds numbers for corrugated airfoil [29, 41]. Vargas et al. [41] noticed that a higher gliding ratio is obtained because of the reduction in drag coefficient due to negative shear produced by the trapped vortices. This study was conducted at Reynolds numbers ranging from 500 to $1 \times 10^{4}$ for angles of attack $0^{\circ}-10^{\circ}$.

Kim et al. [13] found that although the corrugations increases the lift coefficient, they do not affect the drag coefficient values. They conducted the study for $\operatorname{Re}=150-1400$ and angles of attack $0^{\circ}-40^{\circ}$ and found that the Reynolds number has little effect on the aerodynamic performance. Levy and Seifert [19] observed that the flow separated from the corrugations reattaches at the aftupper arc region of the corrugated airfoil which reduces the drag coefficient as well as vortex intensity in the wake. They carried out numerical simulations for Reynolds number range $2000-8000$ and angle of attack $0^{\circ}-10^{\circ}$. Levy and Seifert [18] noted that the vortex shedding process accelerates the reattachment process thereby reducing the drag coefficient. They also reported that a reduction in geometric parameters such as rear arc and trailing edge or rear cavity depth reduces the vorticity magnitude which results in a reduction of drag coefficient. Barnes and Visbal [3] suggested that the local pressure forces in the corrugated airfoil not only reduces the drag coefficient but also enhances the lift coefficient values.

Insects which fly in low Reynolds number range of less than 10,000 have corrugated wing cross sections. Several earlier studies [2, 40,42] have investigated the flow around a live dragonfly. While these studies have provided important insights about the flight of dragonflies, they could not shed enough light on the mechanism responsible behind the secret of their flight. Studies on corrugated airfoils can address this void to an extent. The instantaneous data is not well discussed in the previous studies and therefore available explanations on the physical significance of the trapped vortices are not sufficient to elucidate the high aerodynamic performance of a corrugated airfoil.

In this context, we investigate the underlying flow physics of a rigid corrugated hydrofoil by conducting detailed particle image velocimetry (PIV)-based measurements and force analysis. We have set aside the complexity of dragonfly wing being flexible and three-dimensional; rather the focus is on the effect of corrugations on the surrounding flow. The present results are relevant to gliding (but not to flapping) flights. This study is an extension of Sooraj and Agrawal [36] where the detailed flow dynamics is discussed. The vortex interactions and movements are associated with the range of angle of attack and Reynolds number. Wakeling and Ellington [42] and Levy and Seifert [19] suggested that many insects and small birds fly in the low Reynolds number regime $(\operatorname{Re}<20,000)$. Therefore, the present work covers the entire range of Reynolds number in which the dragonfly fly $(\operatorname{Re}=280-11,700)$, for angles of attack (a) of $0^{\circ}, \pm 5^{\circ}, \pm 10^{\circ}$ and $\pm 15^{\circ}$. The wake velocity profiles are examined to get better understanding about the force variations. The advantages and disadvantages of using positive and negative angles of attack are also discussed. We propose a new hypothesis based on the formation of vortices in the corrugation valleys because of the geometry. We correlate the data of vortex merging to instantaneous lift coefficient variation and also hypothesize the role of travelling waves formed on the hydrofoil by these trapped vortices in reducing drag. The slip effect causes drag reduction as well as lift enhancement thereby uncovering the secret of dragonfly flight.

\section{Materials and methods}

The experimental technique employed in this work along with the procedure for data reduction and uncertainty analysis are detailed in this section.

\subsection{Experimental setup}

The PIV experiments are conducted in a closed-loop water tunnel. The working section of the tunnel has $400 \times 400 \mathrm{~mm}^{2}$ cross-section and a length of $1500 \mathrm{~mm}$ [37]. The corrugated hydrofoil model is made of a rigid acrylic sheet (Fig. 1). This hydrofoil has a span of $400 \mathrm{~mm}$, chord of $50 \mathrm{~mm}$, and thickness of $2 \mathrm{~mm}$ [36] and both ends are attached to the walls during PIV experiments to avoid any three-dimensional effect. The coordinates given by Murphy and Hu [29] is used for the design of the corrugated hydrofoil. The corrugated shape is the cross section of 
Fig. 1 a Schematic of a corrugated hydrofoil. Definition of angle of attack: $\mathbf{b}$ positive and c negative. Note that for the purpose of these experiments, the hydrofoil was inclined in the same direction but with the top and bottom surfaces interchanged

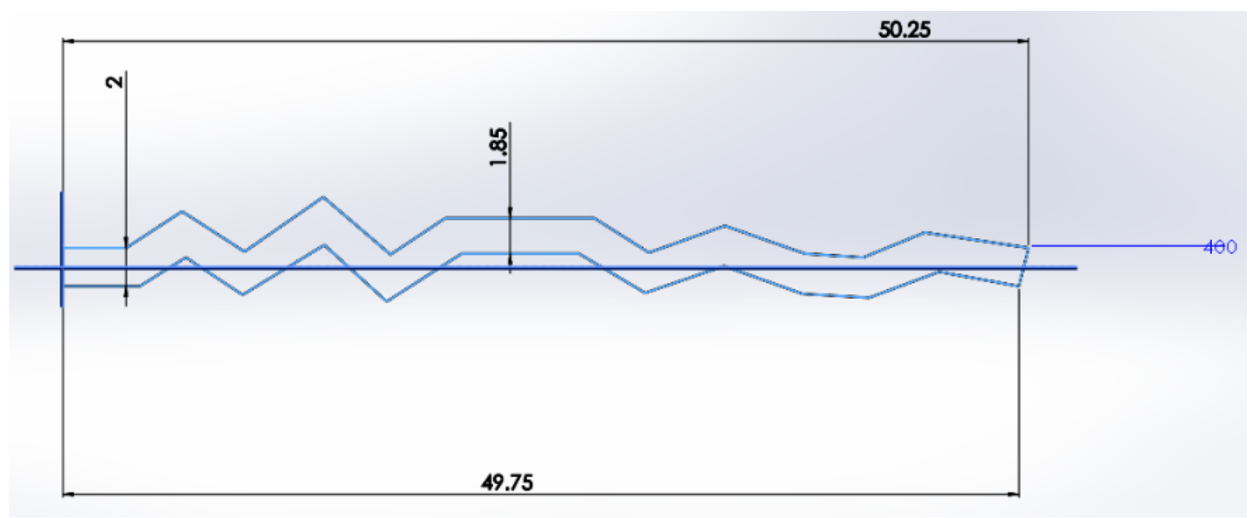

(a)

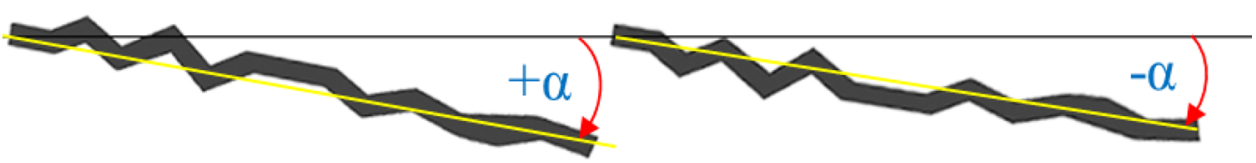

(b)

(c) midspan of a dragonfly wing [11]. The positive and negative angles of attack are defined as shown in Fig. 1b, c. The Reynolds number for the corrugated hydrofoil is in the range of 280-11,700.

The flow velocity varies from 5 to $200 \mathrm{~mm} / \mathrm{s}$. The maximum uncertainty in the mean velocity is estimated to be $\pm 2.7 \%$. The uncertainty in root mean square (r.m.s) values of freestream and lateral velocities are estimated and they are 3.8 and $3.4 \%$ respectively. Effect of seven different angles of attack $\left(0^{\circ}, \pm 5^{\circ}, \pm 10^{\circ}\right.$ and $\left.\pm 15^{\circ}\right)$ is studied covering the range where the aerodynamic performance is excellent as reported in the previous studies. Glass particle of size $8-10 \mu \mathrm{m}$ and specific gravity of 1.1 is used as seeding particle. These glass particles are illuminated using double-pulse Nd:YAG lasers (Beamtech, China; wavelength $532 \mathrm{~nm}$, energy: $200 \mathrm{~mJ} /$ pulse, repetition rate: $15 \mathrm{~Hz}$ ). Seeding particles having are used for the experiments. The particles trace the flow well since the Stokes number for the particle is $2.7 \times 10^{-4}$. The images are captured using a CCD camera (PCO Pixefly, Germany; image size: $1392 \times 1024$ pixels) at a frequency of $5 \mathrm{~Hz}$.

The time delay for a pair of images is varied from 2 to $65 \mathrm{~ms}$ based on the flow speed. The vector spacing is $1.4 \mathrm{~mm}$. There are about $5418(63 \times 86)$ vectors identified in the field. A mirror has been used during the experimentation to illuminate both sides of the hydrofoil and avoid regions of shadow in the view frame. An interrogation window of size of $32 \times 32$ pixels with $50 \%$ overlap is used for analyzing the images. A total of 1500 image pairs have been obtained for each case, which was found adequate for the convergence of the r.m.s. velocity. The images are analyzed using a Matlab based PIV Software PIVlab 1.35, using cross-correlation method [39]. The deviant vectors are identified by comparing a vector with its neighboring velocity vectors. A scatter plot was used to find any such outliers. The percentage of rejected vectors from each frame is less than 4\%. Additional details about our water tunnel and PIV facilities can be found in Khan et al. [12] and Sooraj et al. [35].

\subsection{Force measurement}

A highly sensitive three component load cell (Kistler MiniDyn 9256C1) which can measure up to $\pm 250 \mathrm{~N}$ force with a resolution of $0.002 \mathrm{~N}$, including charge amplifiers (Kistler 5012A) is employed to measure the lift and the drag forces. The schematic of the force measurement is shown in Fig. 2. Dynamic and static calibration of the load cell is carried out [27] and the data is acquired using data acquisition (NI DAQ) system. The data is taken at $2 \mathrm{kHz}$. The high response, resolution and high linearity in the load input/output relation of the dynamometer helps to obtain accurate data. To avoid other disturbances like water tunnel vibrations, if any, the data is taken at two different conditions during the measurement; i.e., without flow and with flow. The force measured without flow is then subtracted from the latter. The pivot is positioned at the mid of the holder containing 
Fig. 2 Schematic of force measurement system
Mating plate

between support and

load cell

\section{Load cell}

Coupling between load cell and airfoil

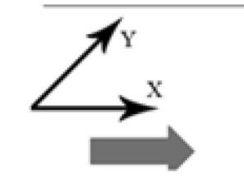

Flow direction

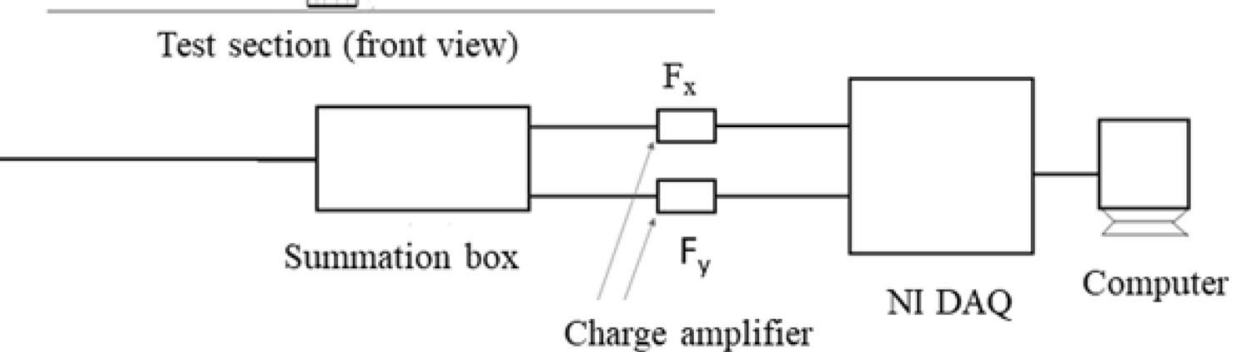

the corrugated hydrofoil, which approximately is the centre of the mass.

\subsection{Uncertainty analysis}

The uncertainty in velocity measurement is estimated based on the four typically employed factors [14, 32, 43]: uncertainty related to equipments employed in the measurement, uncertainty in the ability of particles to track the flow, uncertainty in statistics due to sampling, and uncertainty in data processing. The uncertainty with calibration, magnification, timing accuracy, and CCD recording are combined to obtain the total uncertainty with the image recording system. Stokes' drag law [32] is used to estimate the particle lag. The uncertainty in sampling is estimated by measuring the random measurement error in the sample data using the random error sampling theory. The size of a particle, number of particles in a window, particle image displacement, the effect of background noise and effect of displacement gradients are considered to estimate the uncertainty in processing the data. The standard uncertainty propagation method is employed to combine the uncertainties from individual factors into the overall uncertainty. This procedure suggested that the maximum uncertainty in the mean velocity measurement in the present measurements is $\pm 2.4 \%[35,44]$. Uncertainty in the lift and the drag coefficients are also quantified and presented later in this report.

\section{Wake dynamics}

The variation of streamwise velocity along the crossstream direction at different axial locations are examined and discussed. The results are presented for the Reynolds number range of $280-11,700(280,540,940,2100,3230$, 6700 and 11,700$)$ and angles of attack $-15^{\circ}-15^{\circ}\left( \pm 15^{\circ}\right.$, $\left.\pm 10^{\circ}, \pm 5^{\circ}, 0^{\circ}\right)$. The effect of geometry, angle of attack and Reynolds number can be identified using the wake parameters. The data has been appropriately normalized before plotting. Towards this, we have normalized the streamwise and cross-stream distances by the chord length $(C)$ to obtain $X$ and $Y$ respectively. The free stream velocity $\left(u_{\infty}\right)$ is used as the characteristic velocity for normalizing streamwise velocity $(u)$ and lateral velocity $(v)$ to $U$ and $V$ respectively. The normalized vorticity is obtained by multiplying vorticity by chord length and dividing by the free stream velocity.

\subsection{Instantaneous flow field}

Examining instantaneous flow field is important in understanding the flow behaviour. Instantaneous vorticity field around the corrugated profile is shown in Fig. 3. The effects of angle of attack and Reynolds number are evident from Fig. 3. A virtual hydrofoil profile is obtained when angle of attack $\geq \pm 10^{\circ}$ for $\operatorname{Re} \leq 2100$. Sooraj and Agrawal [36] obtained the virtual hydrofoil when angle of attack is $\pm 5^{\circ}$ 
(a)

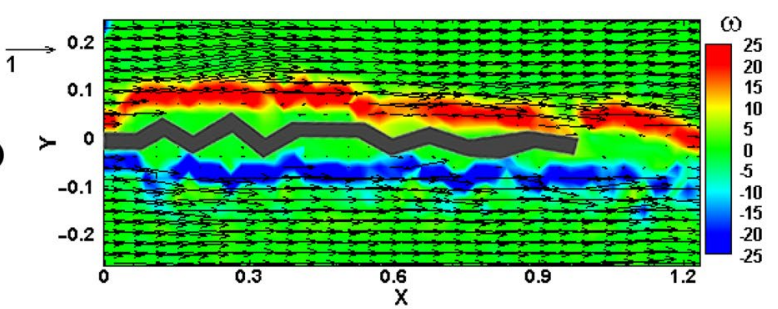

(b)
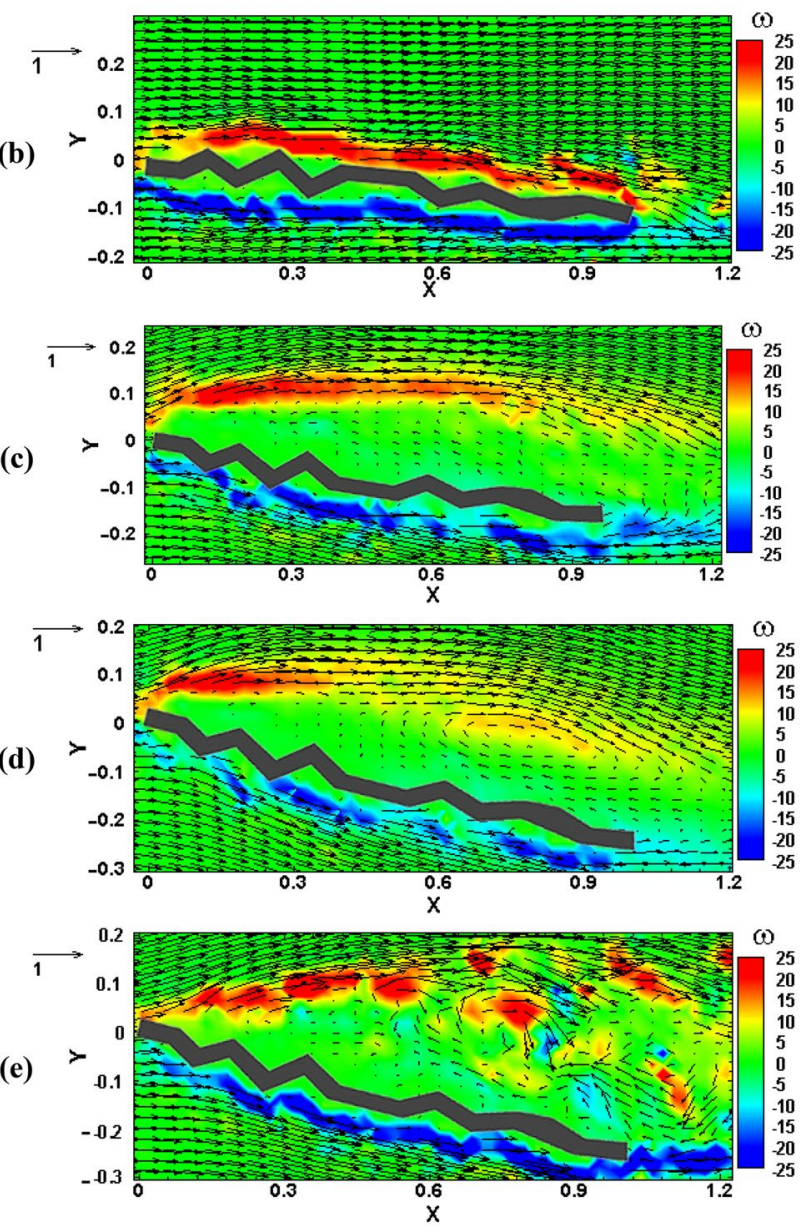

Fig. 3 Instantaneous vorticity contours at a $\operatorname{Re}=11,700, a=0^{\circ}$; b $\operatorname{Re}=11,700, a=5^{\circ} ; \mathbf{c} \operatorname{Re}=940, a=-10^{\circ} ; \mathbf{d} \operatorname{Re}=520, a=-15^{\circ} ;$ e $\operatorname{Re}=6700, a=-15^{\circ}$. The reference vector depicts the magnitude of free stream flow

and $\operatorname{Re} \leq 11,700$. In this section, the flow parameters which leads to the formation of virtual airfoil is discussed.

There are differences in the size and number of trapped vortices because of the asymmetry in the corrugation as the angle of attack changes the sign. The shear layer formed around the corrugated sheet is attached to the surface for angles of attack $0^{\circ}$ and $+5^{\circ}$ (Fig. 3a, b). The shear layer around the corrugated hydrofoil at negative angles of attack is found to be away from the surface consequent to the formation of larger vortices in the corrugations. The corrugated sheet therefore acts as a virtual hydrofoil as explained by Murphy and $\mathrm{Hu}$ [29].

Vortices are trapped in the corrugation and shedding of vortices from one corrugation to another is also observed, which is elaborated further in a later section. For angles of attack $\pm 10^{\circ}$ and $\pm 15^{\circ}$, we observe that the virtual hydrofoil shape is attained at low Reynolds numbers $(\operatorname{Re}<1000)$. Some evidence of this can be seen from the instantaneous images (Fig. 3c, d). Beyond Reynolds number 2100 the wake behind the corrugated sheet is similar to that of the bluff bodies, suggesting that the corrugated sheet cannot maintain the virtual hydrofoil shape at angles of attack $\pm 10^{\circ}$ and $\pm 15^{\circ}$ (Fig. 3e). However, for angles of attack $\pm 5^{\circ}$, the virtual hydrofoil shape is maintained even at $\operatorname{Re}=11,700$. Hence, there is a strong dependence of the flow around a corrugated hydrofoil on the angle of attack and Reynolds number. Another intriguing effect of the virtual hydrofoil shape is seen on the force coefficient values as explained in detail in Sect. 4 below.

\subsection{Streamwise velocity variation}

Time averaged velocity profiles at different streamwise locations in the wake are plotted for four different Reynolds numbers and seven different angles of attack in Figs. 4 and 5. The profiles are plotted along the transverse direction. The wake experiences a momentum deficit due to the presence of the corrugated sheet leading to a deficit in the velocity.

\section{(a) Positive angle of attack}

The velocity variation in the streamwise direction for positive angles of attack is shown in Fig. 4 a. The velocity deficit for the angle of attack $0^{\circ}$ is comparatively lesser than that at higher angles of attack at $X=1.5$. Also, the wake width is smaller for the angle of attack $0^{\circ}$ at all streamwise locations plotted in Fig. 4a. Both these observations suggest that the momentum deficit will be larger for higher angles of attack compared to $a=0^{\circ}$. However, changing the angle of attack from $5^{\circ}$ to $10^{\circ}$ or $15^{\circ}$ does not change the velocity deficit or the wake width significantly until $\operatorname{Re}=2100$. The wake width at the angle of attack $15^{\circ}$ is higher than that at other angles of attack at all streamwise locations. These results are consistent with the variation of the drag coefficient shown later.

It is found that the velocity profiles are having the maximum deficit near the trailing edge $(X=1.5)$ and the velocity values recover towards $X=2$ because of momentum conservation. Except at $R e=280$ for angles of attack $0^{\circ}-15^{\circ}, 70-80 \%$ of the velocity deficit recovers by $X=3$. Interestingly, the velocity deficit recovery for the flow around a corrugated hydrofoil for angle of attack $15^{\circ}$ 
Fig. 4 Velocity variation along the streamwise directions at a $\operatorname{Re}=280 ; \mathbf{b} \operatorname{Re}=2100$ and $\mathbf{c}$ $\mathrm{Re}=6700$ for positive angles of attack

Fig. 5 Velocity variation along the streamwise directions at a $\operatorname{Re}=280 ; \mathbf{b} \operatorname{Re}=2100$ and $\mathbf{c}$ $\operatorname{Re}=11,700$ for negative angles of attack

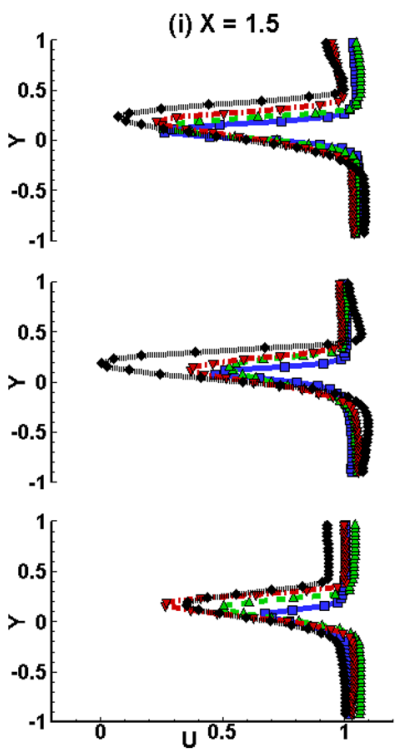

(ii) $X=2$

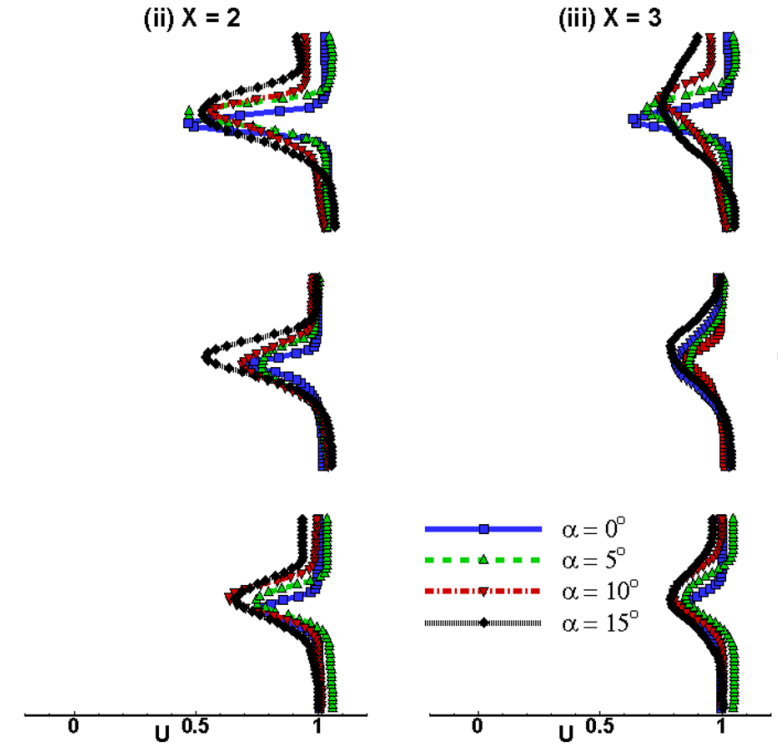

(iii) $\mathrm{X}=3$

(a)

(b)

(c)

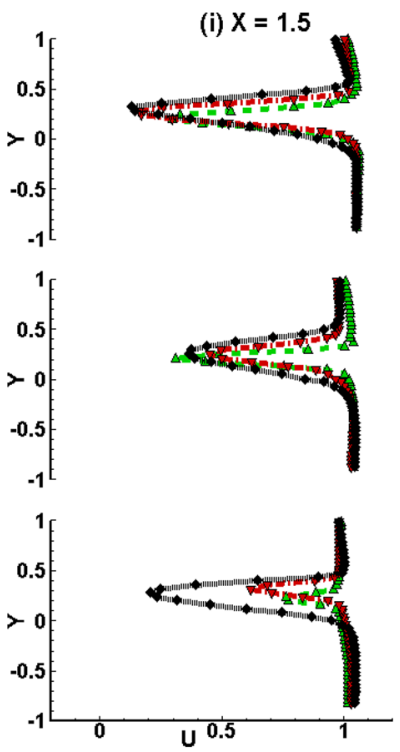

(ii) $X=2$
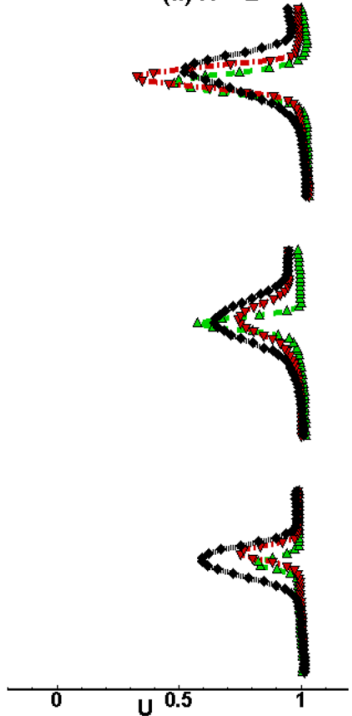

(iii) $X=3$

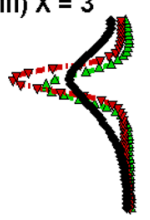

(a)

(b)

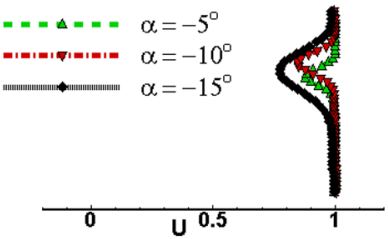

is faster than that at $10^{\circ}$ and $5^{\circ}$ at $R e=280$. Unlike bluff body wakes, formation of large vortex bubble is absent in the wake. This is evident from the positive values of the streamwise velocity.

The rate of recovery of velocity deficit at angles of attack $15^{\circ}$ and $10^{\circ}$ are comparatively higher than $0^{\circ}$ and $5^{\circ}$ at Reynolds numbers 280 and 2100 (Fig. 4a, b). Angle of attack $5^{\circ}$ experiences a faster velocity deficit recovery at $\mathrm{Re}=6700$. The velocity deficit increases for angles of attack $10^{\circ}$ and $15^{\circ}$ as the Reynolds number increases to 6700 .

(b) Negative angle of attack

The velocity profiles for the flow past a corrugated hydrofoil at negative angles of attack are shown in Fig. 5.
Interestingly, $-15^{\circ}$ angle of attack is showing excellent rate of recovery in velocity deficit at $\mathrm{Re}=280$ (Fig. 5a). As the Reynolds number increases to 2100 , angle of attack $-10^{\circ}$ recovers the maximum amount of velocity deficit in comparison to the other angles of attack until $X=2$ (Fig. 5b). A further increase in the Reynolds number results in a better performance at the angle of attack $-5^{\circ}$.

The velocity recovery is from $10 \%$ of the streamwise velocity at $X=1.5-70 \%$ of the streamwise velocity at $X=3$ for the angle of attack $-15^{\circ}$, which is much higher than the angles of attack $-10^{\circ}$ and $-5^{\circ}$. Similarly, a faster rate of velocity recovery is evident for the angle of attack $-5^{\circ}$ at $R e=2100$ as the streamwise distance increases from $X=1.5$ to $X=3$. The momentum loss is relatively less for the angle 
of attack $-5^{\circ}$ at $\operatorname{Re}=11,700$ as seen from Fig. $5 c$, where $60 \%$ of the velocity is recovered at $X=1.5$ itself.

The vortices formed in the corrugation aids the formation of a streamlined shape at low Reynolds numbers for angles of attack $\pm 10^{\circ}$ and $\pm 15^{\circ}$ as shown in Fig. 3c, d. Therefore, the velocity gain is more for $\pm 15^{\circ}$ at $\operatorname{Re}=280$. Similarly, the angles of attack $\pm 10^{\circ}$ are having an early velocity recovery at $\operatorname{Re}=520$ as well as $\operatorname{Re}=940$. Beyond $\operatorname{Re}=2100$, the angles of attack $0^{\circ}$ and $\pm 5^{\circ}$ have lesser velocity deficit compared to the higher angles of attack.

Based on this momentum recovery data, it can be said that the hydrodynamic performance of the corrugated hydrofoil at lower Reynolds numbers $(\operatorname{Re}<1000)$ will be better at angles of attack $-10^{\circ}$ and $-15^{\circ}$. At higher Reynolds numbers, $-5^{\circ}$ angle of attack is superior to the other angles of attack $\left(-10^{\circ}\right.$ and $\left.-15^{\circ}\right)$.

\subsection{Correlation between vortex number and instantaneous lift}

Instantaneous force coefficients and the associated flow fields are very important in the study of swimming or flying animals, as any unbalanced lateral force has to be balanced by some other mechanisms on the body (e.g. movement of the pectoral fins when the caudal fin flaps in fishes) Accordingly, in this section we draw a correlation between the instantaneous flow field at $\operatorname{Re}=2100$ (Fig. 6) and $\mathrm{Re}=3230$ (Fig. 7) for different time steps and the corresponding lift coefficient values (Fig. 8). The lift coefficient is calculated using Eq. 2 provided in Sect. 4.1.

The instantaneous lift coefficient is seen to depend on two primary events: first, formation of vortices in the valleys and second is the merging of vortices formed in the adjoining valleys. Both these events change the number of vortices formed on the corrugated hydrofoil. These events are presented separately below at $\operatorname{Re}=3230$ (Fig. 7) and 2100 (Fig. 6), respectively.

A pair of vortices located with their axes parallel or almost parallel merge when they have the same orientation and the distance between them is less than a certain critical value $[8,15,23,25]$. Even though the dynamical problem is different from Meunier et al. [25], the vortices formed on the corrugated surface satisfy these conditions. Merging of vortices has been found to be important in different contexts as decay of two and three-dimensional turbulent flows [26]; here we examine the effect of vortex merging on the variation of lift coefficient.

In the present analysis of a corrugated surface, merging mainly occurs between the vortices in the 2 nd and 3 rd valleys for $\mathrm{Re}=2100$ as shown in Fig. 6 . At higher Reynolds numbers, merging continues further downstream and occurs even between vortices in the $3 \mathrm{rd}$ and 4 th valleys. These are based on the data from several successive frames. The tendency to merge is high near the leading edge of the corrugated hydrofoil because the frequency of vortex shedding from the 2 nd valley is relatively higher than that from the $3 \mathrm{rd}$ valley. The time for the merging of two vortices decreases as the Reynolds number increases.

Figure 6 presents a sequence of events leading to the merging of the vortices in the adjoining valleys. Notice that the vortex formed in the 2 nd valley has a larger core compared to the vortex in the 1st valley. The vortex size gradually increases due to the viscous effects, i.e., the vorticity of the upstream vortex is gradually but continuously fed to the downstream vortex. The external flow along with the viscous effect triggers the merging. Merging of the vortices results in a bigger vortex on the corrugated surface, which is seen to reduce the lift coefficient (Fig. 8b). That is, merging of vortices reduces the performance of the corrugated hydrofoil. The lift coefficient exhibits a local maximum during the first phase of merging where the vortex pair looks like a dumbbell; this is consistent with the observation of Meunier et al. [26] and explained further by them. The lift coefficient values start decreasing as the distance between the vortex cores decreases beyond a critical distance.

Next, we present evidence for an increase in the lift coefficient with an increase in the number of vortices. The instantaneous lift coefficient data is calculated from PIV data using Eq. 2 presented below. The instantaneous lift coefficient presented in Fig. 8 is therefore synchronized with the corresponding velocity field in Fig. 7. Figure 8a, corresponding to the events in Fig. 7, shows that as the vortices start forming on the upper surface, the lift coefficient increases. The reduction in the value of the lift coefficient at $t=0.4$ is due to the premature state of rolling up of the fluid mass. Such incomplete and weak vortices are apparently not capable of imparting enough acceleration to the flow around the virtual hydrofoil. However, with time the vortices formed in the valleys become stronger (as is evident from Fig. 7b-d), have reasonable size, and produce higher lift (Fig. 8a). The lift coefficient value also increases as the number of vortices on the top surface increases. The above results show the strong correlation between the number of vortices and the value of the instantaneous lift coefficient.

Similar trend in the lift coefficient value is observed for higher angles of attack $\left( \pm 10^{\circ}\right.$ and $\left.\pm 15^{\circ}\right)$ at Reynolds numbers 280,540 and 940 as the number of vortices increases on the upper surface.

\section{Variation in mean force coefficients}

The flow behaviour as noted above should get reflected in the variation of their force coefficients. Accordingly, the variations in the mean drag coefficient, the mean lift coefficient, and the gliding ratio with respect to Reynolds number and 
Fig. 6 Instantaneous streamline plots at $R e=2100$ and $a=-5^{\circ}$ showing merging of vortices near a corrugated hydrofoil

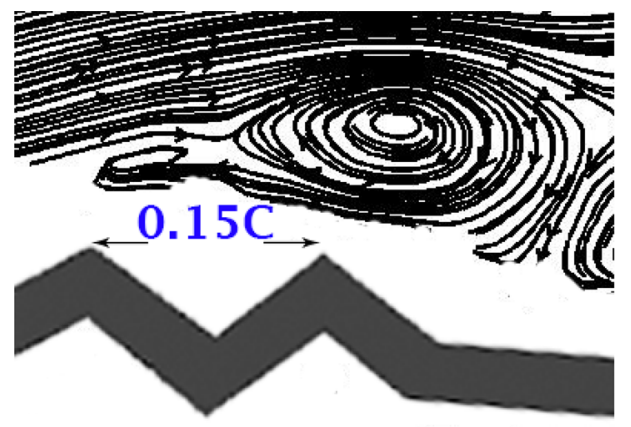

(a) $\mathrm{t}=\mathbf{0 . 0}$

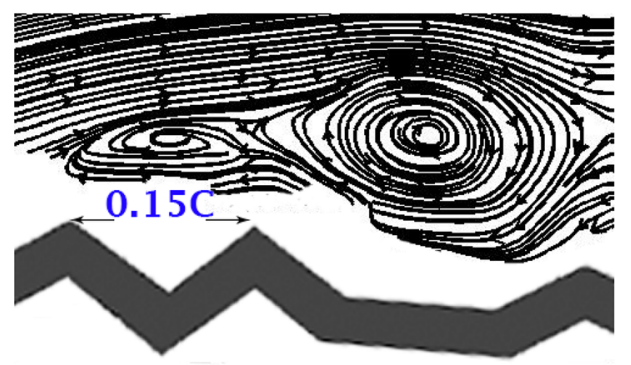

(c) $\mathrm{t}=\mathbf{0 . 2 5 6}$

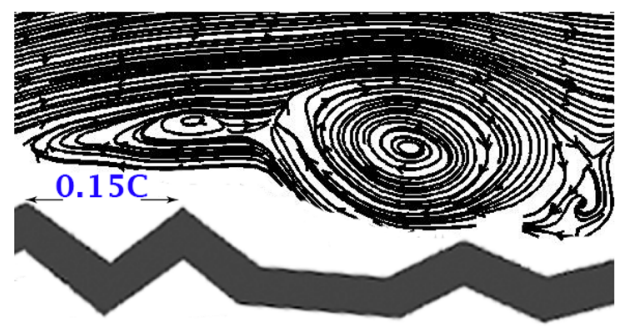

(e) $\mathbf{t}=\mathbf{0 . 5 1 2}$

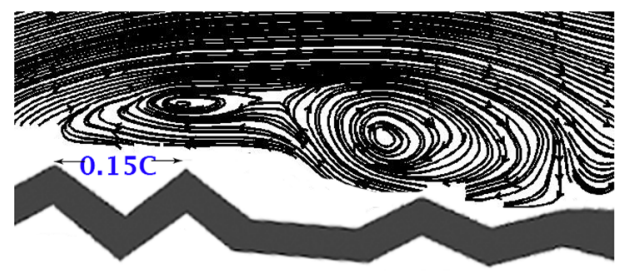

(g) $\mathrm{t}=\mathbf{0 . 7 6 8}$

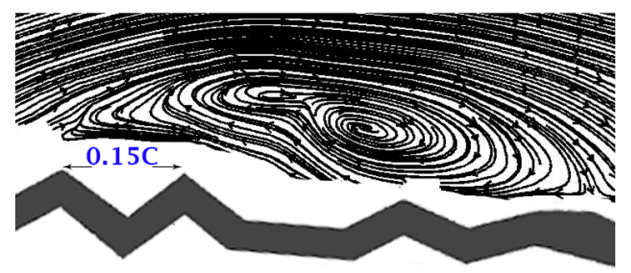

(i) $\quad t=1.024$

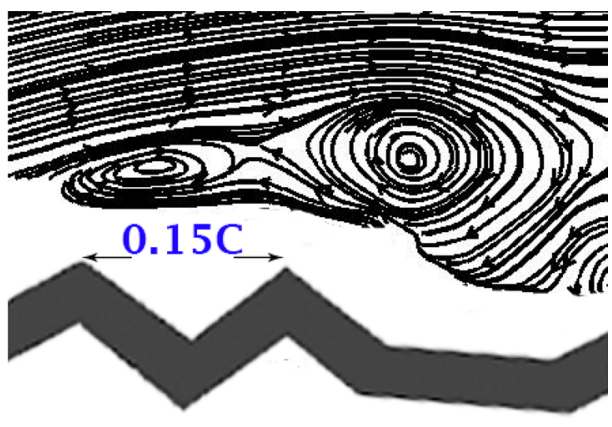

(b) $\mathrm{t}=\mathbf{0 . 1 2 8}$

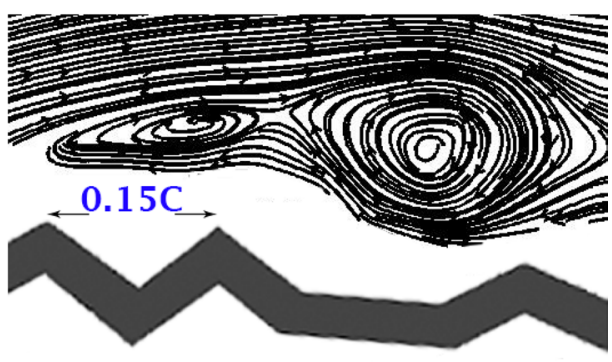

(d) $\mathrm{t}=\mathbf{0 . 3 8 4}$

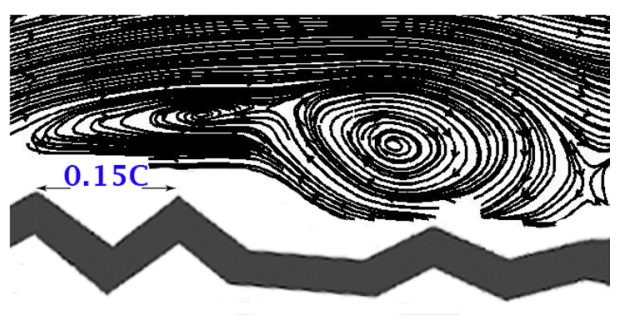

(f) $\mathbf{t}=0.64$

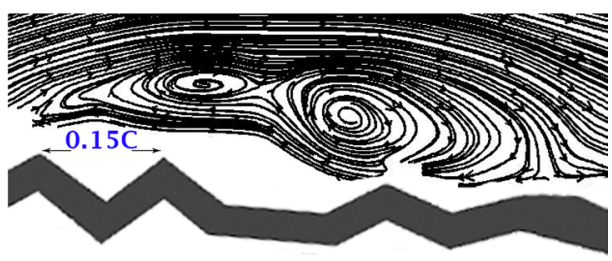

(h) $\mathbf{t}=\mathbf{0 . 8 9 6}$

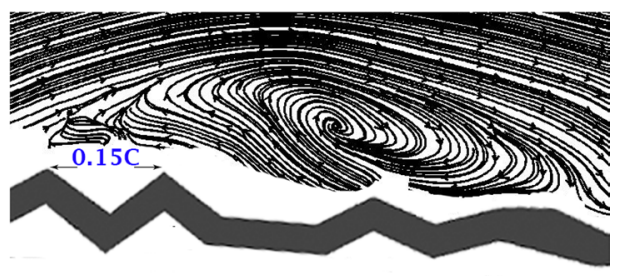

(j) $\mathrm{t}=1.152$ 

line plots at $\operatorname{Re}=3230$ and $a=-5^{\circ}$
Fig. 7 Instantaneous stream-

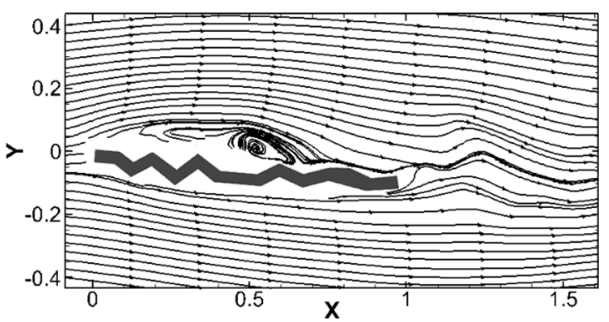

(a) $t=0$

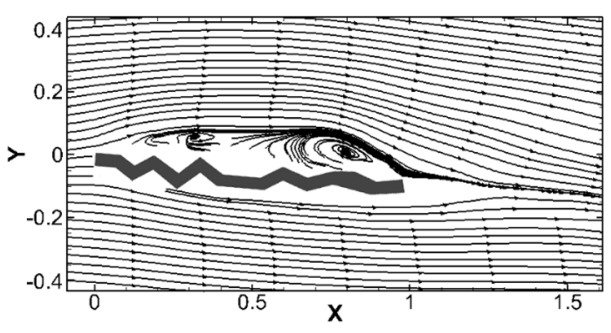

(c) $\mathrm{t}=0.4$

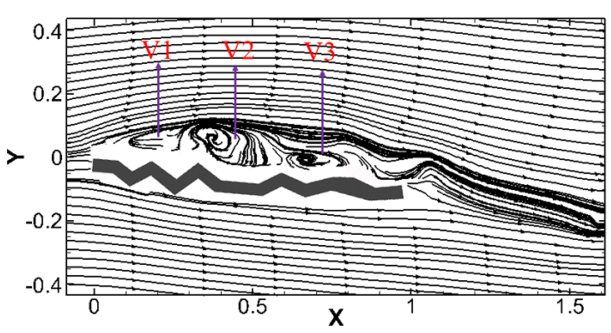

(e) $\mathrm{t}=0.8$

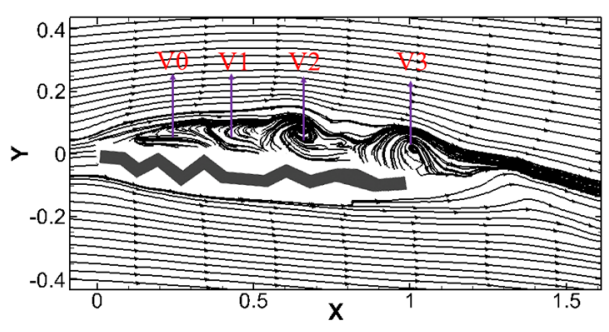

(g) $t=1.2$

Fig. 8 Instantaneous lift coefficient $\left(C_{L}\right)$ corresponding to a Fig. 6 at $\operatorname{Re}=2100, a=-5^{\circ}$ and b Fig. 7 at $\operatorname{Re}=3230, a=-5^{\circ}$. The mean lift coefficient (obtained over a large number of cycles) is also plotted for comparison

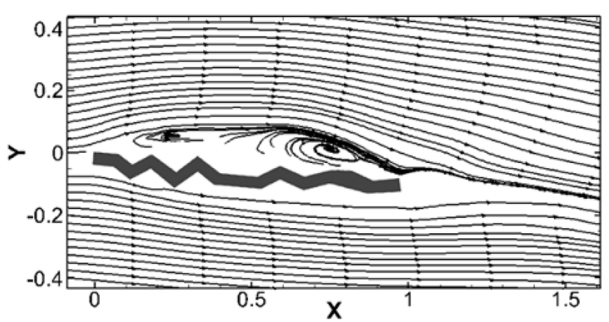

(b) $t=0.2$

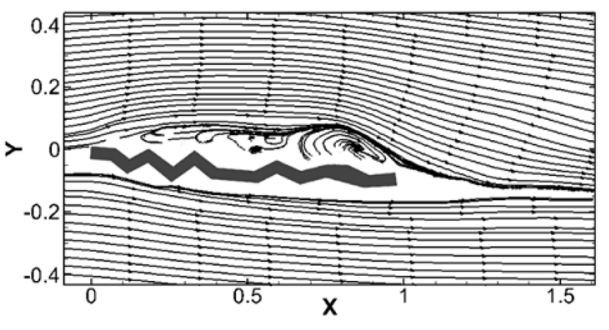

(d) $\mathrm{t}=0.6$

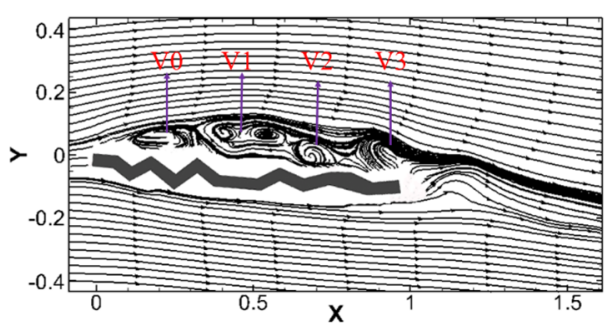

(f) $t=1$

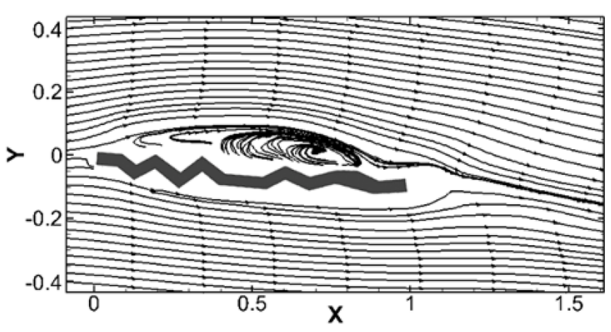

(i) $\mathrm{t}=\mathbf{2 . 4}$

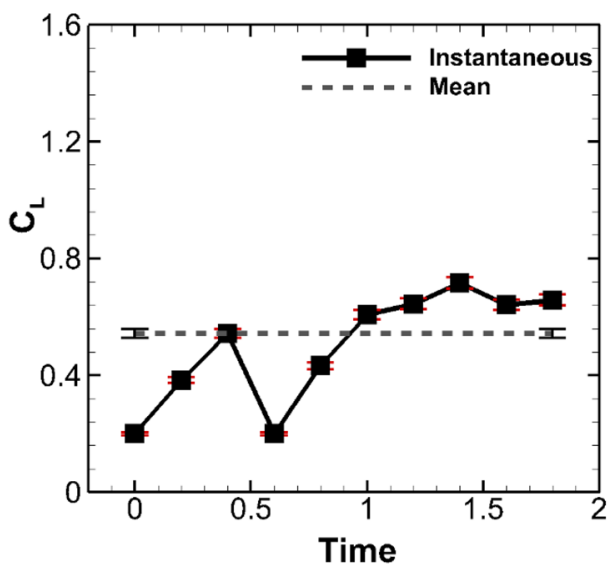

(b)

(a) 
angle of attack are examined in this section. The force coefficient data is obtained by using both the load cell measurement and the PIV data with the wake survey method and the values are compared. Seven different angles of attack $\left(15^{\circ}, 10^{\circ}, 5^{\circ}, 0^{\circ},-5^{\circ},-10^{\circ},-15^{\circ}\right)$ and seven different Reynolds numbers ranging from 280 to 11,700 are studied.

\subsection{Comparison between load cell measurement and wake survey method}

The force coefficients are obtained using mean velocity fields. The modified wake survey method proposed by Bohl and Koochesfahani [4] is used here to calculate the drag coefficient values which incorporates the relative closeness of the control surface to the body. Also, the formulation appropriately considers the fluctuations in the velocity component and the lateral momentum exchange. Based on this approach, the mean drag is written as:
As mentioned above, the overall uncertainty in drag coefficient and lift coefficient is obtained by combining the uncertainty in the individual parameters (in Eqs. 1, 2 and 3) using the uncertainty propagation method, and found to be $\pm 4.4 \%$ and $\pm 3.8 \%$ respectively. Note that Eqs. 1 and 3 involve integration, and the process of integration tends to reduce the uncertainty. Therefore, the actual value of uncertainty in the force coefficient is likely to be smaller than the aforementioned estimates. A separate sensitivity analysis, where the effect of the position of the control surface on the values of the lift coefficients (required in Eq. 3), is also performed. We found that the lift coefficient values converge beyond a downstream distance of $0.7 \mathrm{C}$ and a lateral distance of $0.5 \mathrm{C}$ ( $\mathrm{C}$ is the chord length); since the presented results employ these distances, the present results are deemed to be well converged.

The drag and the lift coefficient values calculated for the angle of attack $+5^{\circ}$ from the load cell measurement

$C_{D}=\frac{2}{C} \int_{-H}^{+H}\left[\frac{u_{\text {avg }}}{u_{\infty}}\left(\frac{u_{\text {avg }}}{u_{\infty}}-1\right)+\varepsilon\left(\frac{u_{\text {avg }}}{u_{\infty}}-1\right)+\left(\frac{u_{r m s}}{u_{\infty}}\right)^{2}-\left(\frac{v_{r m s}}{u_{\infty}}\right)^{2}+\frac{1}{2}\left(1-\frac{u_{0}^{2}}{u_{\infty}^{2}}\right)\right] d y$

where $C$ is the chord length; $2 H$ is the height of the control surface; $u_{\text {avg }}$ is the mean velocity; $u_{\infty}$ is the freestream velocity; $u_{o}$ is the outlet velocity; $u_{r m s}$ is the fluctuating component of streamwise velocity and $v_{r m s}$ is the fluctuating component of lateral velocity. The effective velocity $\varepsilon$ is much smaller than unity. The values of $\partial \mathrm{v}_{\mathrm{rms}}^{2} / \partial \mathrm{y}$ term is dominant in the transverse momentum equation. The mean transverse velocity and the streamwise gradient of Reynolds stresses are found to be negligible.

The Kutta-Joukowski theorem is used to determine the value of the lift coefficient [7]. For this, the lift force is calculated as:

$L^{\prime}=\rho_{\infty} u_{\infty} \Gamma$

where $\rho_{\infty}$ and $\Gamma$ are respectively the density of water and the circulation. The circulation required in the above formula is calculated from the velocity field obtained by PIV. From the definition of circulation, we have

$\Gamma=\oint \boldsymbol{v} \cdot d \boldsymbol{l}$

where $\boldsymbol{V}$ is velocity of the flow field and $d \boldsymbol{l}$ is the elemental length (boldface indicates vector quantity). The line integration can be completed for any closed path around the body. The lift force can be calculated for either the mean or the instantaneous flow field. The lift coefficient is finally obtained by normalizing the value of the lift force per unit depth by $\frac{1}{2} \rho u_{\infty}^{2} C$. and the wake survey methods are compared with the results obtained by Barnes and Visbal [3] and Vargas et al. [41] as shown in Fig. 9. Evidently, the force coefficient values obtained from both the load cell measurement and the wake survey method are matching very well with the results obtained by Barnes and Visbal [3] and Vargas et al. [41]. Notice that the calculated values of lift and drag coefficients using PIV data and load cell measurements compare within $5 \%$, which is well within the uncertainty of these measurements.

\subsection{Variation in drag}

The drag coefficient on a corrugated hydrofoil decreases as the Reynolds number increases for angles of attack $\pm 5^{\circ}$ as shown in Fig. 10. The drag coefficient value increases with an increase in the angle of attack. Vargas et al. [41] also showed that the drag coefficient value increases as the angle of attack increases from $0^{\circ}$ to $5^{\circ}$. Interestingly, the drag coefficient decreases when the angle of attack increases from $5^{\circ}$ to $15^{\circ}$ at $R e=280$. As the Reynolds number increases to 940 , the drag coefficient for the angle of attack $15^{\circ}$ displays a relative increase so as to exceed the corresponding drag value at angle of attack $5^{\circ}$. Whereas, the drag value for the angle of attack $15^{\circ}$ exhibits similar behaviour with respect to $5^{\circ}$ much later as the Reynolds number approaches 2100 . Even though, the drag values for the positive and negative angles of attack are the same at $10^{\circ}$ and $15^{\circ}$, there is a noticeable difference 

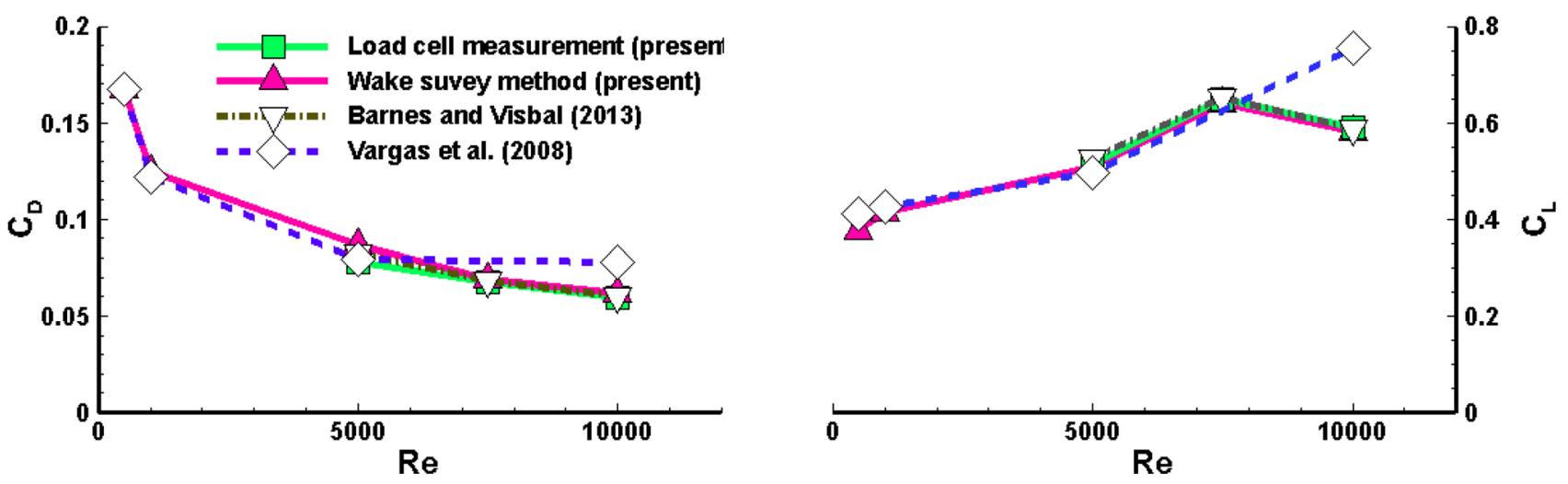

Fig. 9 Comparison of force coefficient values $\left(C_{D}\right.$ : drag coefficient, $C_{L}$ : lift coefficient) with the literature $+5^{\circ}$
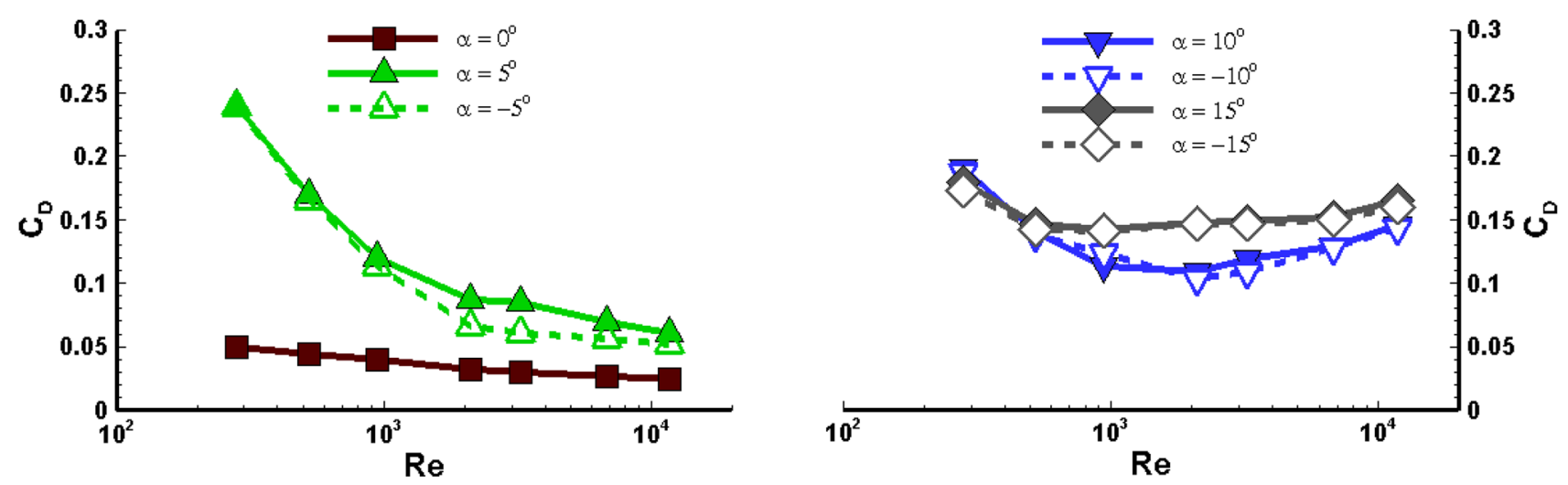

Fig. 10 Drag coefficient $\left(C_{D}\right)$ values calculated for different angles of attack and Reynolds numbers

at the angle of attack $5^{\circ}$ especially for Reynolds numbers 2100 and 3230 . Shedding of the trapped vortices formed in the corrugations is observed for $a=-5^{\circ}$ from $R e=2100$ onwards, while shedding of trapped vortices is observed at $\operatorname{Re}=11,700$ for $a=+5^{\circ}$.

The reduction in the drag can be explained by considering the virtual hydrofoil that is formed over the corrugated hydrofoil. This virtual hydrofoil has a non-zero velocity at its boundary and also has a large acceleration on the top surface of the hydrofoil due to the trapped vortices. These effects lead to a low value of the drag coefficient for the corrugated hydrofoil. The above argument about the flow remaining attached to a corrugated hydrofoil leading to a reduction in the drag is reinforced by considering the data at angles of attack $10^{\circ}$ and $15^{\circ}$.

The present observations can be further understood by considering the contribution of the individual components - shear drag and pressure drag —on the overall drag. The pressure drag increases substantially upon flow separation, which in turn increases the total drag. But the flow does not separate for low Reynolds numbers in the case of a corrugated hydrofoil, which makes the pressure drag small. The total drag for a corrugated hydrofoil is mainly dependent on the shear drag that is rather small. The 'virtual' slip on a corrugated hydrofoil (discussed in detail further below) reduces the shear drag further.

From these observations it can be concluded that the drag coefficient decreases with an increase in the Reynolds number for $0^{\circ}$ and $5^{\circ}$ angles of attack. Also, the drag coefficient for $10^{\circ}$ and $15^{\circ}$ angles of attack is smaller than $5^{\circ}$ at low Reynolds numbers $(\operatorname{Re}<1000)$ and higher than $5^{\circ}$ at high Reynolds numbers (Re $>1000)$.

\subsection{Variation in lift}

Figure 11 shows that the lift coefficient exhibits a nonmonotonic variation with respect to the Reynolds number. The maximum value of the lift coefficient is achieved at $\operatorname{Re}=6700$ for the angle of attack $5^{\circ}$. As the angle of attack increases to $10^{\circ}$, the maximum value of the lift coefficient is obtained at a lower Reynolds number $(R e=2100)$. The trend in variation of the lift coefficient exhibited by the 

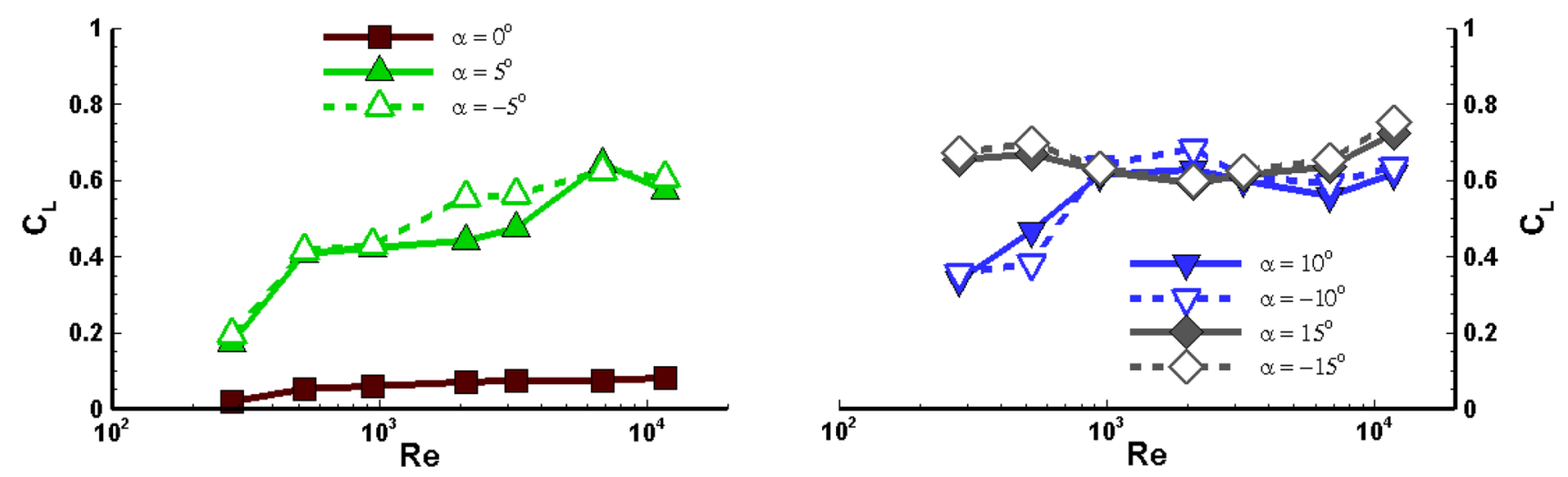

Fig. 11 Lift coefficient $\left(C_{L}\right)$ values calculated for different angles of attack and Reynolds numbers

corrugated hydrofoil is similar to that of a flat plate as the Reynolds number increases. Also, the lift coefficient value for $0^{\circ}$ angle of attack is negligibly small compared to $5^{\circ}$ angle of attack. This was also observed by Vargas et al. [41]. There is a considerable increase in the lift coefficient values at $\operatorname{Re}=2100$ and 3230 for $a=-5^{\circ}$ and no noticeable difference is observed as the sign of the angle of attack changes for other cases.

The variation in the lift coefficient with Reynolds number is mainly dependent on the shape of the virtual hydrofoil and the strength of the trapped vortices, which in turn depends on the Reynolds number. The acceleration of flow on the surface of the virtual hydrofoil alters the pressure distribution and enhances the lift on the corrugated hydrofoil.

In summary, the results show that the lift coefficient increases with an increase in angle of attack from $5^{\circ}$ to $10^{\circ}$ till $R e=2100$ and the first lift peak of the lift coefficient occurs even earlier $(R e=523)$ for the angle of attack $15^{\circ}$. The number and size of the vortices formed on the upper surface of the corrugated hydrofoil and the closeness of the vortices to the corrugated wing determines the amount of lift produced.

\subsection{Variation in gliding ratio}

The variation in the gliding ratio (defined as the ratio of the mean lift to the mean drag) as a function of the Reynolds number and the angle of attack is plotted in Fig. 12. The gliding ratio increases with the Reynolds number at the angle of attack $5^{\circ}$. Large gliding ratio values are observed at the angle of attack $-5^{\circ}$ for $2100<\operatorname{Re}<11,700$. The gliding ratio values are comparatively lesser for the positive angle of attack. The gliding ratio for the angles of attack $10^{\circ}$ and $15^{\circ}$ is higher at lower Reynolds numbers, but smaller at higher Reynolds numbers as compared to the $-5^{\circ}$ angle of attack. This changeover occurs at $\operatorname{Re} \sim 520$ for $a=15^{\circ}$ and $\operatorname{Re} \sim 2100$ for $a=10^{\circ}$. The gliding ratio is comparatively small at $R e=2100$ for the angle of attack $15^{\circ}$ because of a reduction in the lift coefficient value due to the change in the shape of the virtual hydrofoil.
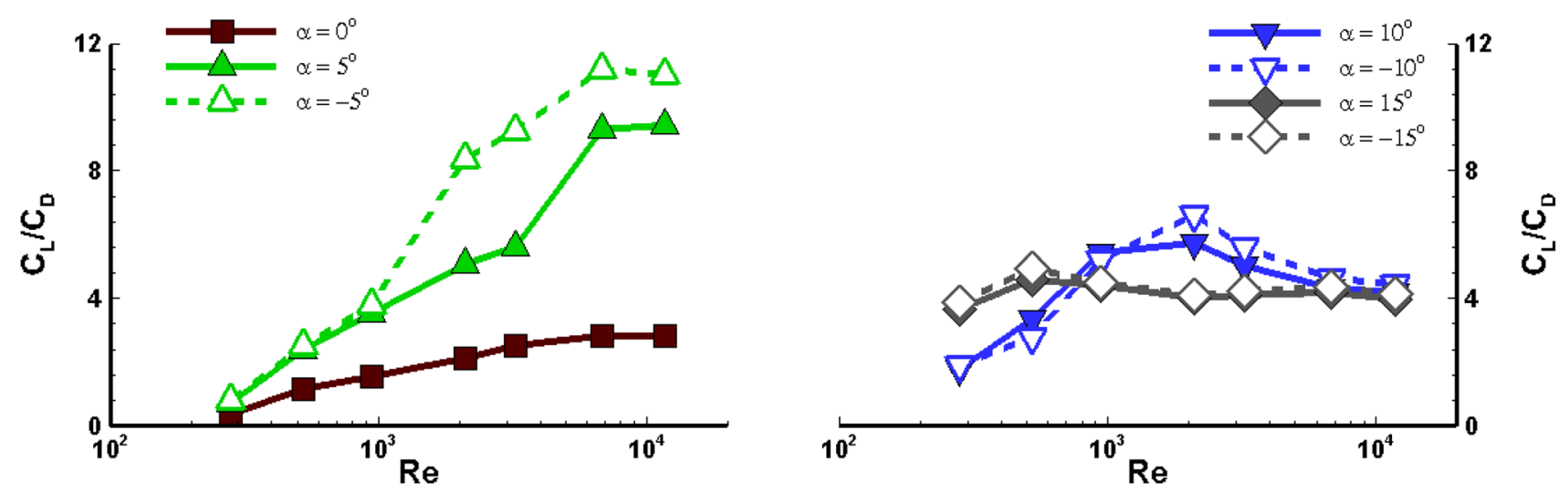

Fig. 12 Gliding ratio values calculated for different angles of attack and Reynolds numbers 
These results explain the hydrodynamic performance of the corrugated hydrofoil at the angles of attack $\pm 5^{\circ}$ and also the advantages of corrugation. The gliding ratio is maximum at $\operatorname{Re}>940$ for $-5^{\circ}$ angle of attack. The results further show how the vortex interactions increase the lift, which helps to improve the gliding ratio. Although the gliding ratio is higher for the negative angles of attack, nature does not prefer the negative angles of attack. The reason for this becomes apparent upon examining Fig. 8 where a large variation in the instantaneous lift coefficient value is seen. This unbalanced lift force would lead to a lateral movement of the dragonfly making it unsuitable for flight from the stability point of view.

\section{Discussion}

In this section, the flow around a dragonfly wing cross section is discussed in detail based on the low drag values and the shedding of vortices from one corrugation to another. The vortices on the lower side are relatively stronger compared to the ones on the upper side which help to increase the lift as explained in Meng et al. [24], which is however not seen with the present data. It is rather argued that the slip effect on the virtual surface is the main reason for the additional lift. The asymmetry in the geometry and asymmetry in the flow help to generate relatively high lift for a corrugated hydrofoil.

As explained in the earlier sections, the vortices formed in the valleys of the corrugation act as a boundary for the virtual hydrofoil surface (Fig. 7). It is also shown that these vortices are continuously shed from one valley to another, forming a moving virtual boundary (Fig. 6). These moving vortices thus function as fluid roller bearings, producing a travelling wave which moves with a definite speed and becomes the moving virtual boundary of the corrugated hydrofoil (Fig. 13). The fluid roller bearing effect explained by Chuijie et al. [5], albeit in a different context, essentially removes the boundary layer with the aid of a travelling wave produced by the flexible wall. In the latter case, the vortices trapped inside the troughs act as the fluid roller bearing. The corrugated hydrofoil with the virtual boundary is similar to a hydrofoil (streamline body) with a slip on the surface. The vortices that act as roller bearings merge with each other due to both convection and the viscous diffusion effects and new vortices form periodically to replace these merged vortices.

The instantaneous lift coefficient values also show that the travelling waves are effective when their wavelength is relatively small. In order to achieve a smaller wavelength, the number of corrugations should be greater. But, an increased number of corrugations reduce the performance owing to vortex shedding. So, there should be an optimized design accommodating both the criteria; smaller wavelength of the travelling wave and the number of corrugations.

An apparent slip is found to be generated on the superhydrophobic surface because of the air-water interface, which affects the flow field irrespective of the shape of the body_bluff, streamline, or flat plate $[1,16,17,28,37]$. The shear layers formed on the superhydrophobic surface are weak as the velocity gradient values are small because
Fig. 13 Schematic of the 2-D flow around a corrugated dragonfly wing

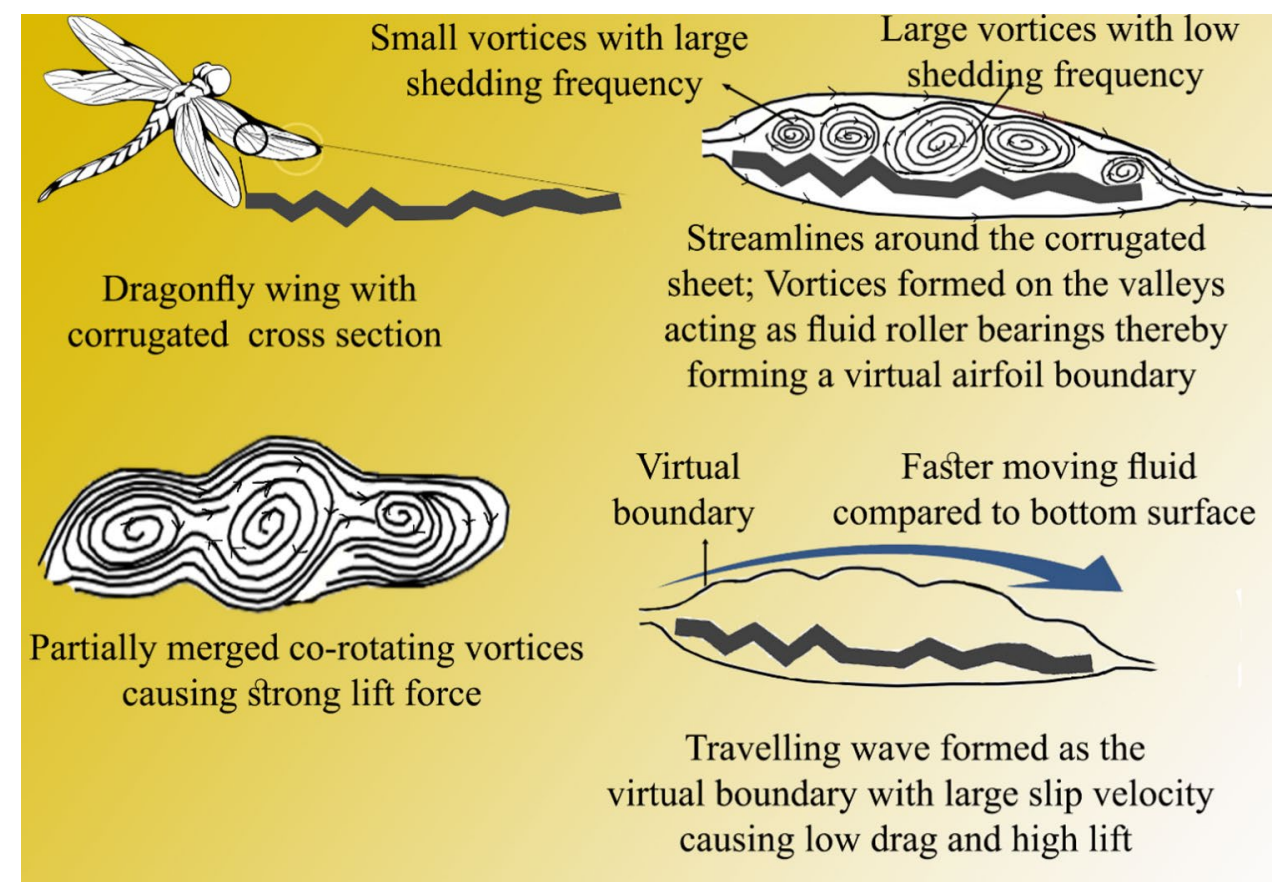


of the partial slip. The weaker shear layers cause a delay in flow separation, low vortex intensity in the wake, early rolling up of vortices, increase in the shedding frequency, and a reduction in the drag coefficient values $[16,17,37]$.

The presence of corrugation demonstrates the effects on flow dynamics (causing slip) similar to that achieved through a superhydrophobic surface modification. This enables to view corrugated hydrofoil as a combination of both shape and surface modification. The above observation is further emphasized in the comparative analysis of the lift and drag coefficients of both the surfaces.

A corrugated hydrofoil exhibits a smaller value of drag coefficient than the superhydrophobic hydrofoil which already exhibits a low drag coefficient value in comparison to an Acrylic hydrofoil. The latter phenomenon is consequent to the partial slip generated on a superhydrophobic surface (Fig. 14a, c). The amount of drag reduction increases as the angle of attack increases. A drag reduction of $36 \%$ is obtained when a corrugated hydrofoil is employed instead of a superhydrophobic hydrofoil at $5^{\circ}$; the reduction increased to $56 \%$ at $15^{\circ}$ for $\operatorname{Re}=6760$ (Fig. 14a). The variation in drag reduction is from 36 to $61 \%$ for $5^{\circ}-15^{\circ}$ when Reynolds number is increased to 11,700 (Fig. 14c).

At the same time, there is an enhancement in the lift coefficient for the corrugated hydrofoil compared to the superhydrophobic hydrofoil. There is an almost fivefold increase in lift coefficient at angle of attack $5^{\circ}$ which reduces to $66 \%$ when the angle of attack is increased to $15^{\circ}$ at $\operatorname{Re}=6760$ (Fig. 14b). The increase in the lift coefficient is 297 and $89 \%$ at $5^{\circ}$ and $15^{\circ}$ respectively, for $\operatorname{Re}=11,700$ (Fig. 14d).

Levy and Seifert [19] proposed a corrugated hydrofoil model based on the cross section of dragonfly wing that has a similar design but the profile is smoother (less corrugated) than the original cross section. Their proposed design has two sharp protrusions near the leading edge followed by a widely-open, smoothly-curved valley and a trailing edge which is a small extension of the valley. Nevertheless, the basic structure is the same as a dragonfly wing cross section. The maximum gliding ratio obtained by the corrugated profile suggested by Levy and Seifert [19] is less than 11 for angle of attack $5^{\circ}$ at $\mathrm{Re}=8000$ while the present study shows a gliding ratio value greater than 11 in the Reynolds number range $6700-11,700$ for angle of attack $-5^{\circ}$. The present results suggest that it is better to have some amount of corrugation instead of a smooth curved valley because the corrugation triggers vortex formation. It is further evident from the instantaneous lift coefficient values that the formation of stronger vortices leads to an enhancement in the value of lift coefficient. Thus, the natural shape of dragonfly wing is better than the proposed shape in the literature.

The time-averaged vorticity contour around the corrugated hydrofoil is shown in Fig. 15. Sooraj and Agrawal [36] reported that the shear layer act as a virtual
Fig. 14 Comparison of drag coefficient and lift coefficient for a corrugated hydrofoil with a superhydrophobic hydrofoil and an acrylic hydrofoil at different angles of attack [37]

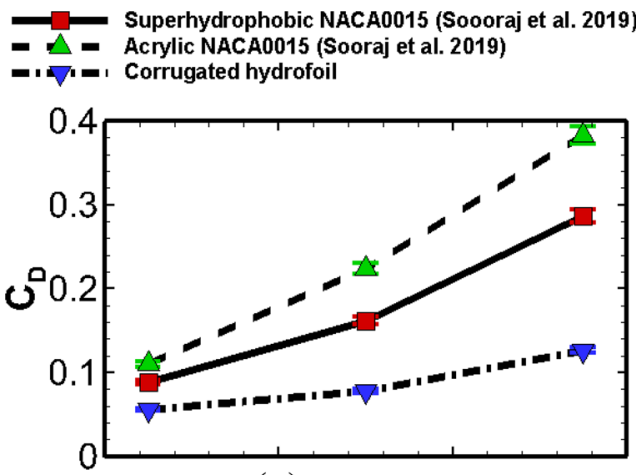

(a) $\operatorname{Re}=6760$

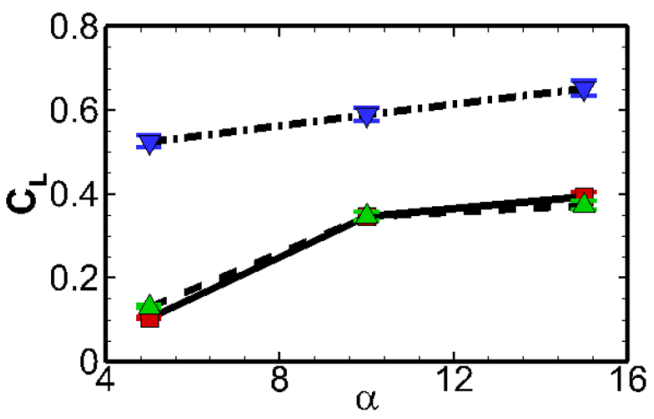

(b) $\operatorname{Re}=6760$

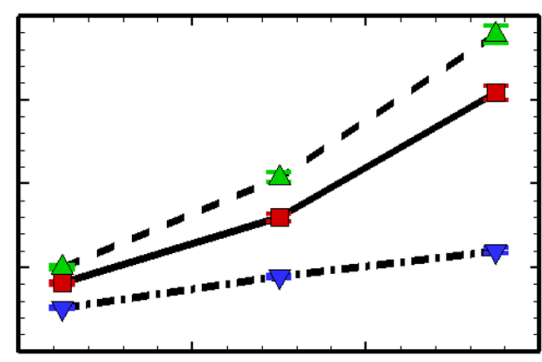

(c) $\operatorname{Re}=11700$

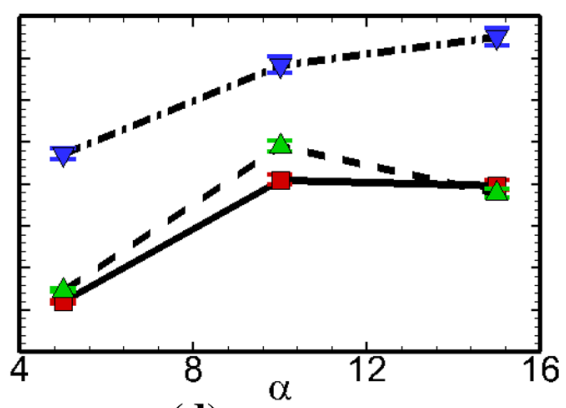

(d) $\operatorname{Re}=11700$

SN Applied Sciences 
hydrofoil surface at angles of attack $\pm 5^{\circ}$ for Reynolds number range 340-11,700. At $\operatorname{Re}=540$, a virtual hydrofoil shape is formed around the corrugated sheet for angles of attack $\pm 10^{\circ}$ and $\pm 15^{\circ}$ (Fig. 15a, c) The formation of a virtual hydrofoil shape results into lower drag compared to $a= \pm 5^{\circ}$ as evident from Fig. 10. The shear layers start moving away from the surface as the Reynolds number increases for angles of attack $\pm 10^{\circ}$ and $\pm 15^{\circ}$ (Fig. 15b, d) which result to an increase in the drag coefficient.

Vargas et al. [41] reported that the influence of shear drag in the total drag is comparatively negligible due to the large regions of negative shear. The negative shear drag is formed because of the trapped vortices in the corrugation valleys which is in line with the observations of Rees [33], Rudolph [35] and Newman et al. [30]. Therefore, the role of viscosity in the total drag is negligibly small. The present study also shows that the drag coefficient value is comparatively lesser for corrugated hydrofoil (Fig. 14). These results substantiate the hypothesis proposed in the present study which suggests that the higher hydrodynamic performance of the corrugated hydrofoil is due to the large 'virtual slip'.

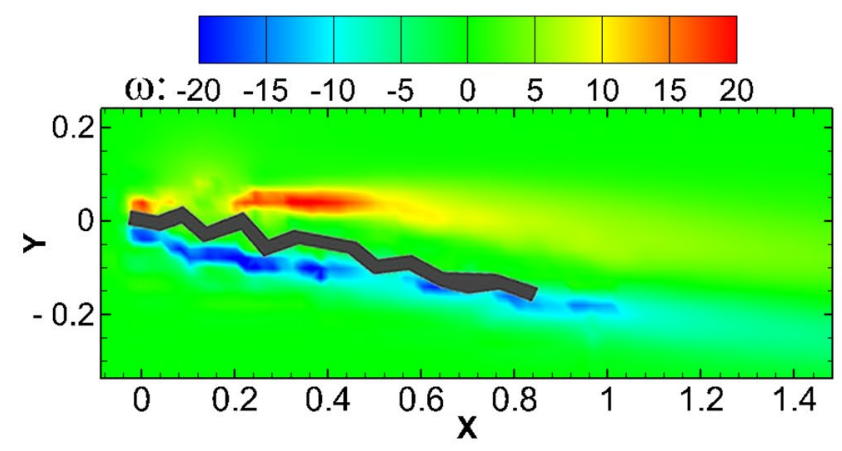

(a)

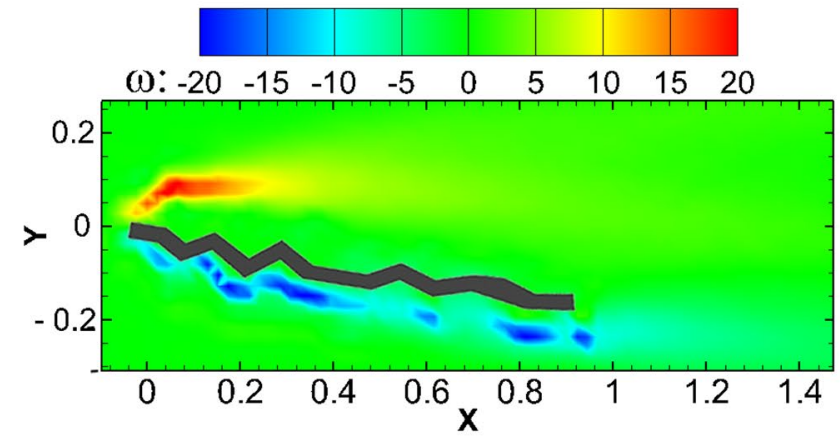

(c)

\section{Conclusion}

An experimental study has been conducted on a corrugated hydrofoil at different Reynolds numbers $(\operatorname{Re}=280-11,700)$ and angles of attack $\left(-15^{\circ}\right.$ to $\left.+15^{\circ}\right)$ using the PIV technique. The study tries to answer why corrugation is required at low Reynolds number and what happens to the flow at these low Reynolds numbers. Instantaneous streamlines, vorticity, lift, drag and gliding ratio are computed and examined thoroughly to understand the flow dynamics.

The hydrodynamic performance of a corrugated hydrofoil is strongly dependent on the Reynolds number and the angle of attack. It is found that the corrugated wing functions as a virtual hydrofoil with the help of the rotating fluid masses formed in the corrugation valleys. The variation in the wake velocity profiles suggest that the corrugated sheet is performing better at high angles of attack $\left( \pm 10^{\circ}\right.$ and $\left.\pm 15^{\circ}\right)$ for low Reynolds numbers $(\operatorname{Re}<1000)$ and beyond $\operatorname{Re}=2100$, the angle of attack $5^{\circ}$ is performing better than the other angles of attack. The growth and movement of the vortices from the top surface largely affect the force coefficients. The lift coefficient increases when relatively large number

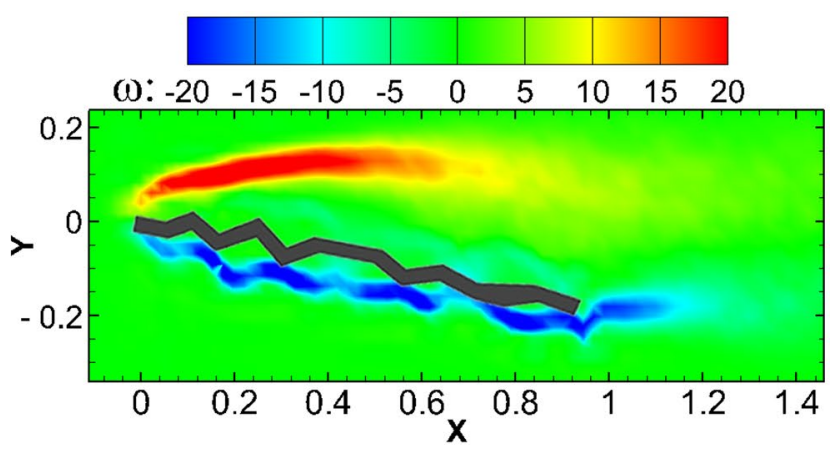

(b)

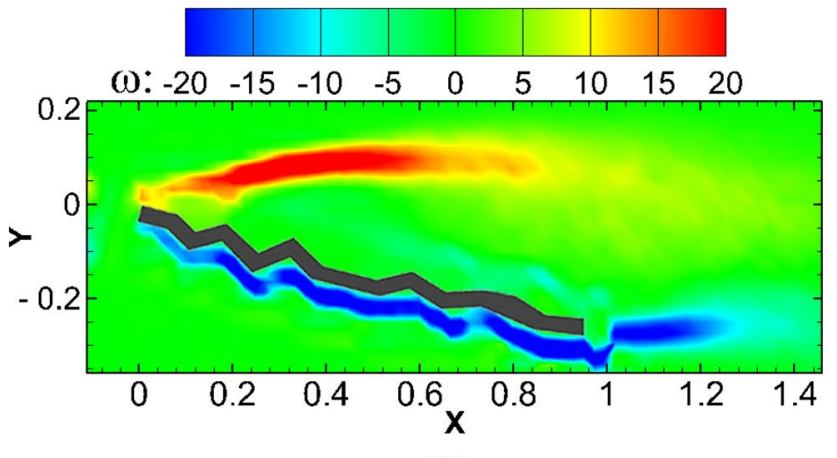

(d)

Fig. 15 Time averaged vorticity contours for $\mathbf{a} a=10^{\circ}, \operatorname{Re}=540 ; \mathbf{b} \mathbf{a}=10^{\circ}, \operatorname{Re}=6760 ; \mathbf{c} a=-15^{\circ}, \operatorname{Re}=540 ; \mathbf{d ~} \mathbf{a}=-15^{\circ}, \operatorname{Re}=6760$ 
of vortices are present on the top surface. The vorticity strength reduces considerably when vortices shed into the wake. The mean lift and the mean drag coefficients vary depending on the formation of virtual hydrofoil. The maximum gliding ratio is obtained for angles of attack $\pm 5^{\circ}$. Even though the gliding ratio is higher for $-5^{\circ},+5^{\circ}$ is preferred by nature since the fluctuations in the lateral force coefficient is higher for $-5^{\circ}$.

The arrangement of corrugation valleys is argued to be very important for a corrugated wing for Reynolds number less than 10,000 , for obtaining favorable vortex arrangement. The vortex interaction, vortex shedding and the shape of the virtual hydrofoil profile are strongly dependent upon the size and shape of the corrugations. Corrugation valleys are narrower near the leading edge and become wider towards the trailing edge. The trapped vortices act as roller bearings, which create a travelling wave on the virtual hydrofoil profile that eliminates the boundary layer formation, and thereby induces a very large slip on the virtual surface. The slip on the surface causes a higher lift and a low drag, which results in better hydrodynamic performance of a corrugated hydrofoil. The merging of co-rotating vortices on the corrugated surface at higher Reynolds numbers is an important phenomenon. These partially-merged vortices make the travelling wave more effective and thereby produce a greater lift. These results and insights should aid in better design of micro air vehicles.

\section{Compliance with ethical standards}

Conflict of interest The author(s) declare that they have no competing interests.

\section{References}

1. Aljallis E, Sarshar MA, Datla R, Sikka V, Jones A, Choi CH (2013) Experimental study of skin friction drag reduction on superhydrophobic flat plates in high Reynolds number boundary layer flow. Phys Fluids 25(2):025103

2. Azuma A, Watanabe T (1988) Flight performance of a dragonfly. J Exp Biol 137(1):221-252

3. Barnes CJ, Visbal MR (2013) Numerical exploration of the origin of aerodynamic enhancements in [low-Reynolds number] corrugated airfoils. Phys Fluids 25(11):115106

4. Bohl DG, Koochesfahani MM (2009) MTV measurements of the vortical field in the wake of an airfoil oscillating at high reduced frequency. J Fluid Mech 620:63-88

5. Chuijie W, Yanqiong X, Jiezhi W (2003) "Fluid roller bearing" effect and flow control. Acta Mech Sin 19:476-484

6. Corbet PS (1983) A biology of dragonflies. Facsimile reprint. EW Classey Ltd., Faringdon, XVI

7. Dickinson $\mathrm{MH}$ (1996) Unsteady mechanisms of force generation in aquatic and aerial locomotion. Am Zool 36:537-554
8. Dritschel DG (1995) A general theory for two-dimensional vortex interactions. J Fluid Mech 293:269-303

9. Heinrich B (1993) Heat managers (Book reviews: The hotblooded insects. Strategies and mechanisms of thermoregulation). Science 260:1155-1156

10. Hu H, Tamai M (2008) Bioinspired corrugated airfoil at low Reynolds numbers. J Aircr 45(6):2068-2077

11. Kesel $A B$ (2000) Aerodynamic characteristics of dragonfly wing sections compared with technical aerofoils. J Exp Biol 203(20):3125-3135

12. Khan MH, Sooraj P, Sharma A, Agrawal A (2018) Flow around a cube for Reynolds numbers between 500 and 55000. Exp Therm Fluid Sci 93:257-271

13. Kim WK, Ko JH, Park HC, Byun D (2009) Effects of corrugation of the dragonfly wing on gliding performance. J Theor Biol 260(4):523-530

14. Lazar E, DeBlauw B, Glumac N, Dutton C, Elliott G (2010) A practical approach to PIV uncertainty analysis. AIAA Pap 4355:30

15. Le Dizes S, Verga A (2002) Viscous interactions of two co-rotating vortices before merging. J Fluid Mech 467:389-410

16. Lee J, Kim H, Park H (2018) Effects of superhydrophobic surfaces on the flow around an NACA0012 hydrofoil at low Reynolds numbers. Exp Fluids 59(7):111

17. Lee J, Kim H, Park H (2018) Effects of superhydrophobic surfaces on the flow around an NACA0012 hydrofoil at low Reynolds numbers. Exp Fluids 59:111

18. Levy DE, Seifert A (2010) Parameter study of simplified dragonfly airfoil geometry at Reynolds number of 6000 . J Theor Biol 266(4):691-702

19. Levy DE, Seifert A (2009) Simplified dragonfly airfoil aerodynamics at Reynolds numbers below 8000. Phys Fluids 21(7):071901

20. May M (1995) Dependence of flight behavior and heat production on air temperature in the green darner dragonfly Anax junius (Odonata: Aeshnidae). J Exp Biol 198(11):2385-2392

21. May ML (1978) Thermal adaptations of dragonflies. Odonatologica $7(1): 27-47$

22. May ML (1976) Thermoregulation and adaptation to temperature in dragonflies (Odonata: Anisoptera). Ecol Monogr 46(1):1-32

23. Melander MV, Zabusky NJ, McWilliams JC (1988) Symmetric vortex merger in two dimensions: causes and conditions. J Fluid Mech 195:303-340

24. Meng XG, Xu L, Sun M (2011) Aerodynamic effects of corrugation in flapping insect wings in hovering flight. J Exp Biol 214(3):432-444

25. Meunier P, Ehrenstein U, Leweke T, Rossi M (2002) A merging criterion for two-dimensional co-rotating vortices. Phys Fluids 14:2757-2766

26. Meunier P, Le Dizes S, Leweke T (2005) Physics of vortex merging. C R Phys 6:431-450

27. Mittal RK, Kulkarni SS, Singh R (2018) Characterization of lubrication sensitivity on dynamic stability in high-speed micromilling of Ti-6Al-4V via a novel numerical scheme. Int J Mech Sci 142:51-65

28. Muralidhar P, Ferrer N, Daniello R, Rothstein JP (2011) Influence of slip on the flow past superhydrophobic circular cylinders. J Fluid Mech 680:459-476

29. Murphy JT, Hu H (2010) An experimental study of a bio-inspired corrugated airfoil for micro air vehicle applications. Exp Fluids 49(2):531-546

30. Newman BG, Savage SB, Schouella D (1977) Model tests on a wing section of an Aeschna dragonfly. In Pedley TJ (ed) Scale effects in animal locomotion. Academic Press, London, New York, pp 445-477 
31. Polcyn DM (1994) Thermoregulation during summer activity in Mojave Desert dragonflies (Odonata: Anisoptera). Funct Ecol 8:441-449

32. Raffel M, Willert CE, Scarano F, Kähler CJ, Wereley ST, Kompenhans J (2001) Particle image velocimetry: a practical guide. Springer, Berlin

33. Rees CJ (1975) Form and function in corrugated insect wings. Nature 256:200-203

34. Rudolph R (1978) Aerodynamic properties of Libellula quadrimaculata L. (Anisoptera: Libellulidae), and the flow around smooth and corrugated wing section models during gliding flight. Odonatologica 7:49-58

35. Sooraj P, Agrawal A, Sharma A (2018) Measurement of drag coefficient for an elliptical cylinder. J Energy Environ Sustain 5:1-7

36. Sooraj P, Agrawal A (2018) Flow around a corrugated airfoil. J Flow Vis Image Process 25(3-4):145-162

37. Sooraj P, Jain S, Agrawal A (2019) Flow over hydrofoils with varying hydrophobicity. Exp Therm Fluid Sci 102:479-492

38. Sunada S, Sakaguchi A, Kawachi K (1997) Airfoil section characteristics at a low Reynolds number. J Fluids Eng 119(1):129-135

39. Thielicke W, Stamhuis E (2014) PIVlab-towards user-friendly, affordable and accurate digital particle image velocimetry in MATLAB. J Open Res Softw 2:e30
40. Thomas AL, Taylor GK, Srygley RB, Nudds RL, Bomphrey RJ (2004) Dragonfly flight: free-flight and tethered flow visualizations reveal a diverse array of unsteady lift-generating mechanisms, controlled primarily via angle of attack. J Exp Biol 207(24):4299-4323

41. Vargas A, Mittal R, Dong H (2008) A computational study of the aerodynamic performance of a dragonfly wing section in gliding flight. Bioinspir Biomim 3(2):026004

42. Wakeling JM, Ellington CP (1997) Dragonfly flight. I. Gliding flight and steady-state aerodynamic forces. J Exp Biol 200(3):543-556

43. Wang L, Hejcik J, Sunden B (2007) PIV measurement of separated flow in a square channel with streamwise periodic ribs on one wall. J Fluids Eng 129:834-841

44. Yadav H, Srivastava A, Agrawal A (2016) Characterization of pulsating submerged jet-a particle image velocimetry study. J Therm Sci Eng Appl 8:011014

Publisher's Note Springer Nature remains neutral with regard to jurisdictional claims in published maps and institutional affiliations. 